\title{
ANÁLISE QUALITATIVA DE SÓLIDOS ELASTOPLÁSTICOS SOB DEFORMAÇÃO FINITA UTILIZANDO ELEMENTOS DE BARRA BI- ARTICULADOS E 3D 2D
}

\section{Qualitative Analysis of Elastoplastic Solids under Finite Deformation using Bi-Articulated Bar Elements 2D and 3D}

\section{Wiliam Taylor Matias Silva', Wellington Andrade da Silva' ${ }^{2}$, Marcus Alexandre Noronha de Brito ${ }^{3}$}

Recebido em 16 de fevereiro de 2016; recebido para revisão em 08 de julho de 2016; aceito em 10 de agosto de 2016; disponível on-line em 03 de setembro de 2016.

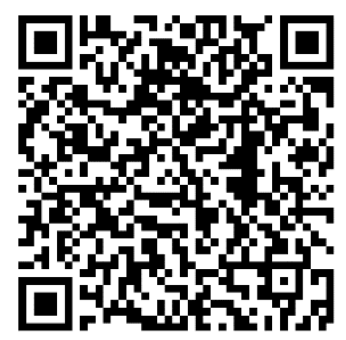

PALAVRAS CHAVE:

Formulação lagrangeana total;

Deformações elastoplásticas finitas; Elementos de barra biarticulados.

\section{KEYWORDS:}

Total langrangian formulation; Elastoplastic strains finite; Bi-Articulated bar elements.

RESUMO: Neste trabalho apresenta-se uma descrição Lagrangeana Total para retratar sólidos elastoplásticos sob deformação finita. Discretiza-se estes sólidos com elementos de treliça 2D e 3D com o intuito de obter analiticamente o vetor de forças internas e a matriz de rigidez tangente. Assume-se um modelo constitutivo hiperelástico para o estado uniaxial de tensão-deformação, utilizando a tensão de Kirchhoff e a deformação logarítmica, que formam um par energeticamente conjugado. Para descrever as deformações plásticas finitas utiliza-se a hipótese da decomposição multiplicativa do estiramento uniaxial do elemento de treliça. Por fim, apresentam-se algumas simulações numéricas de sólidos 3D discretizados com elementos de treliça 2D e 3D.

ABSTRACT: This paper presents a Lagrangian Total description to describe elastoplastic solids under finite deformation. These solids are discretized with truss elements $2 \mathrm{D}$ and $3 \mathrm{D}$ aiming to obtain analytically internal force vector and the tangent stiffness matrix. Are assumed a hyperelastic constitutive model for the state of uniaxial stress-strain using the Kirchhoff's stress and logarithmic strain, to form a conjugate pair energetically. To describe the finite plastic deformation using the hypothesis of the multiplicative decomposition of uniaxial stretching of the truss element. Finally, we present some numerical simulations of solid 3D discretized with 2D and 3D truss elements.

* Contato com os autores:

1e-mail: taylor@unb.br (W.T.M. Silva)

Professor Associado da Universidade de Brasília - UnB.

2e-mail: wellington_andrade@ufg.br (W. A. da Silva)

Professor Adjunto da Faculdade de Engenharia - Regional Catalão - Universidade Federal de Goiás.

${ }^{3}$ e-mail: marcusanb@yahoo.com.br (M. A. N. de Brito)

Professor Adjunto do Departamento de Construção Civil do Instituto Federal de Brasília, Campus Samambaia. 


\section{INTRODUÇÃO}

Citar as inúmeras contribuições científicas das últimas décadas dedicadas ao estudo e desenvolvimento do método dos elementos finitos aplicado na análise não-linear da mecânica dos sólidos seria uma tarefa Hercúlea, porém deste fato, salta a vista, a importância e a relevância conquistada pela mecânica computacional em diversos setores de tecnologia de ponta desenvolvidas pelo homem na atualidade. Tornouse quase imperativo, para o avanço da ciência, compreender e simular os fenômenos não-lineares em diversas áreas do conhecimento, tais como, biomecânica, mecânica dos fluídos, geotecnia, mecânica dos sólidos, engenharia de tecidos humanos, etc. $\mathrm{Na}$ área dos métodos numéricos aplicados a engenharia, por exemplo, recomenda-se a leitura das seguintes referências: Crisfield (1991), Crisfield (1997), Simo e Hughes (1998), Belytschko et al (2000), Doyle (2001), Wriggers (2002), Kojić e Bathe (2005), Bonet e Wood (2008), Wriggers (2008), Neto et al (2008), Krenk(2009), Voyiadjis e Woelke (2010), Borst et al. (2012) e Hashiguchi e Yamakawa (2013).

Decorre, daí, a necessidade de difundir os conceitos básicos e fundamentais da análise nãolinear através do método dos elementos finitos, imbuído deste espírito, o trabalho, aqui apresentado, sem maiores pretensões teóricas, tem como objetivo apresentar uma análise teórica e numérica de sólidos elastoplásticos sob deformação finita discretizados com elementos de barra biarticulados 2D ou 3D. Propõe-se utilizar elementos de barra biarticulados por sua simplicidade teórica, o que permite descrever, facilmente, a cinemática do movimento do elemento e obter o vetor de forças internas e a matriz de rigidez tangente analiticamente, que são elementos imprescindíveis para uma análise nãolinear em mecânica dos sólidos. Para simular deformações elásticas finitas, assume-se um modelo constitutivo hiperelástico para o estado uniaxial de tensão-deformação, utilizando a tensão de Kirchhoff e a deformação logarítmica, que formam um par energeticamente conjugado. Para descrever as deformações plásticas finitas utiliza-se a hipótese da decomposição multiplicativa do estiramento uniaxial. Por fim, apresentam-se algumas simulações numéricas de sólidos 2D e 3D discretizados com elementos de treliça 2D e 3D, respectivamente.

\section{DESCRIÇÃO CINEMÁTICA DO ELEMENTO BI- ARTICULADO 3D}

Para expressar as variáveis cinemáticas na configuração indeformada, definida no instante $t=$ 0 , utilizam-se as coordenadas materiais $\left(X_{1}, X_{2}, X_{3}\right)$, enquanto que, na configuração deformada, definida no instante $t$, usam-se as coordenadas espaciais $\left(x_{1}, x_{2}, x_{3}\right)$ conforme se mostra na Figura 1 . No instante $t=0$, o comprimento do elemento, a área da seção transversal e seu volume são dados por $L$, $A$ e $V$, respectivamente. Após o elemento sofrer grandes translações, grandes rotações e deformações finitas ele ocupa a configuração deformada no instante $t$. Neste instante, o comprimento do elemento, a área da seção transversal e o volume são dados por $l, a$ e $v$, respectivamente. $O$ comprimento e a direção do elemento no instante $t$ são expressos, em função das coordenadas $\vec{x}_{a}$ e $\vec{x}_{b}$, como $l=\sqrt{\left(\vec{x}_{b}-\vec{x}_{a}\right) \cdot\left(\vec{x}_{b}-\vec{x}_{a}\right)}$ e $\vec{n}=\frac{1}{l}\left(\vec{x}_{b}-\vec{x}_{a}\right)$, respectivamente. Ainda no instante $t, \vec{u}_{a}$ e $\vec{u}_{b}$ representam deslocamentos incrementais das extremidades do elemento a partir deste intante. Observe que estes deslocamentos não são os deslocamentos entre as configurações indeformada e deformada. Sob a hipótese de que o elemento sofre deformações uniformes, pode-se definir uma medida de deformação a partir do estiramento expresso por $\lambda=\frac{l}{L}$. Por outro lado, a relação entre os volumes do elemento, entre os instantes $t=0 \mathrm{e}$ $t$, é escrita como $J=\frac{v}{V}$. Se o elemento alonga de $d l$ a partir do comprimento atual $l$, a deformação instântanea é definida como $d \varepsilon=\frac{d l}{l}$. Integrando esta deformação instantânea ao longo do elemento entre os instantes $t=0$ e $t$, pode-se definir a deformação logarítmica como $\varepsilon=\ln \lambda=\int_{L}^{l} \frac{d l}{l}$. Considerando-se que as deformações normais ao eixo do elemento bi-articulado sejam iguais em todas as direções, que é o caso de materiais isotrópicos, deduz-se que $J=\lambda^{(1-2 v)}$ ou $v=V\left(\frac{l}{L}\right)^{(1-2 v)}$. A demonstração desta expressão se encontra detalhada no Quadro 1. 


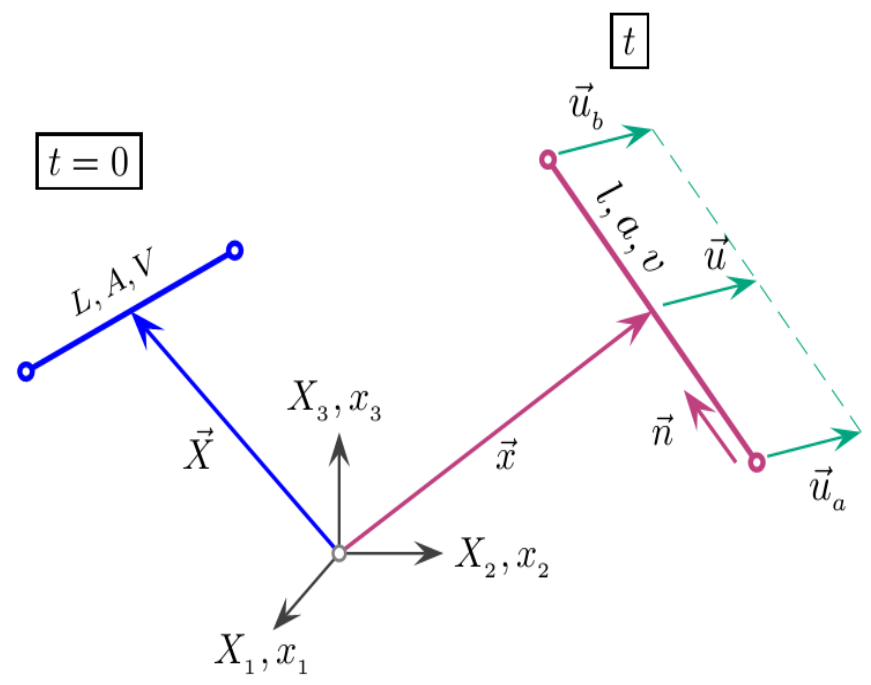

FIGURA 1: Movimento do elemento biarticulado 3D.

FONTE: Próprios autores.

\section{QUADRO 1: Relação entre os volumes do elemento nos instantes $t=0$ e $t$.}
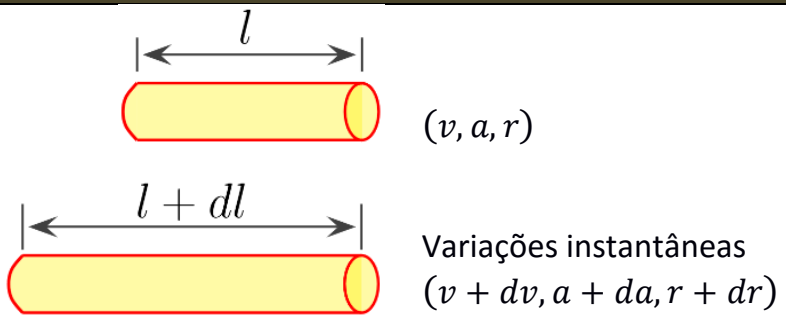

\begin{tabular}{|c|c|}
\hline Volume atual & $v=a l$ \\
\hline Área atual & $a=\pi r^{2}$ \\
\hline Deformação axial instantânea & $d \epsilon=\frac{d l}{l}$ \\
\hline Deformação radial instantânea & $d \epsilon_{r}=\frac{d r}{r}=-v \frac{d l}{l}$ \\
\hline Variação volumétrica instantânea & $d v=a d l+l d a$ \\
\hline Onde & $d a=2 \pi d r=2 a \frac{d r}{r}=-2 a v \frac{d l}{l}$ \\
\hline Assim & $d v=a d l-2 a v d l=(1-2 v) a d l$ \\
\hline ou & $\frac{d v}{v}=(1-2 v) \frac{d l}{l}$ \\
\hline Assumindo que $v=$ cte e integrando chega-se a & $\begin{array}{c}\int_{V}^{v} \frac{d v}{v}=(1-2 v) \int_{L}^{l} \frac{d l}{l} \\
\ln \frac{v}{V}=(1-2 v) \ln \frac{l}{L}\end{array}$ \\
\hline ou & $J=\frac{v}{V}=\left(\frac{l}{L}\right)^{(1-2 v)}=\lambda^{(1-2 v)}$ \\
\hline
\end{tabular}




\subsection{LINEARIZAÇÃO DAS VARIÁVEIS CINEMÁTICAS}

Para obter a matriz de rigidez tangente utilizada na solução de um sistema de equações de equilíbrio não-lineares é necessário linearizar as variáveis cinemáticas do elemento biarticulado. A linearização das variáveis cinemáticas será obtida através da derivada direcional em relação aos deslocamentos incrementais das extremidades do elemento definidos a partir da configuração deformada. A derivada direcional do vetor $l \vec{n}=$ $\vec{x}_{b}-\vec{x}_{a}$, é dada por meio da Equação 1.

$$
\begin{aligned}
D\left(\vec{x}_{b}-\vec{x}_{a}\right)[\vec{u}] & =\left.\frac{d}{d \epsilon}\right|_{\epsilon=0}\left(\vec{x}_{b}+\epsilon \vec{u}_{b}\right. \\
& \left.-\vec{x}_{a}-\epsilon \vec{u}_{a}\right) \\
& =\left(\vec{u}_{b}-\vec{u}_{a}\right)
\end{aligned}
$$

A derivada direcional do comprimento atual $l$ do elemento pode ser obtida mais facilmente notando que $\quad \operatorname{Dl}(\vec{x})[\vec{u}]=2 \operatorname{lDl}(\vec{x})[\vec{u}]$, consequentemente, $\quad D l(\vec{x})[\vec{u}]=\frac{1}{2 l} D l^{2}(\vec{x})[\vec{u}]$. A derivada direcional de $l^{2}$ é dada por meio da Equação 2.

$$
\begin{gathered}
D l^{2}(\vec{x})[\vec{u}]=\left.\frac{d}{d \epsilon}\right|_{\epsilon=0}\left\{\left(\vec{x}_{b}+\epsilon \vec{u}_{b}-\vec{x}_{a}\right.\right. \\
\left.-\epsilon \vec{u}_{a}\right) \\
\cdot\left(\vec{x}_{b}+\epsilon \vec{u}_{b}-\vec{x}_{a}\right. \\
\left.\left.-\epsilon \vec{u}_{a}\right)\right\} \\
=2\left(\vec{x}_{b}-\vec{x}_{a}\right) \cdot\left(\vec{u}_{b}-\vec{u}_{a}\right) \\
=2 l \vec{n} \cdot\left(\vec{u}_{b}-\vec{u}_{a}\right)
\end{gathered}
$$

A partir da qual obtém-se a Equação 3.

$$
\operatorname{Dl}(\vec{x})[\vec{u}]=\vec{n} \cdot\left(\vec{u}_{b}-\vec{u}_{a}\right)
$$

De maneira similar, será útil para derivações futuras, a seguinte relação (Equação 4).

$$
\begin{aligned}
D l^{-1}(\vec{x})[\vec{u}]= & -l^{2} D l(\vec{x})[\vec{u}] \\
& =-l^{-2} \vec{n} \cdot\left(\vec{u}_{b}-\vec{u}_{a}\right)
\end{aligned}
$$

Por último, a derivada direcional da deformação logarítmica, $\varepsilon=\ln \lambda=\ln (l / L)$, é dada pela seguinte expressão

$$
\begin{aligned}
D \varepsilon(\vec{x})[\vec{u}]=D( & \ln l(\vec{x})-\ln L)[\vec{u}] \\
& =\frac{1}{l} D l(\vec{x})[\vec{u}] \\
& =\frac{1}{l} \vec{n} \cdot\left(\vec{u}_{b}-\vec{u}_{a}\right)
\end{aligned}
$$

\section{FORÇAS INTERNAS E MODELO CONSTITUTIVO HIPERELÁSTICO}

Conforme mostra-se na Figura 2, as forças internas $\vec{t}_{a}$ e $\vec{t}_{b}$ são facilmente determinadas em função da tensão verdadeira de Cauchy $\sigma$, da área da seção transversal $a$ e da direção $\vec{n}$ do elemento bi-articulado. Portanto, escrevem-se estas forças como $\vec{t}_{a}=-\sigma a \vec{n}$ e $\vec{t}_{b}=-\sigma a \vec{n}$, e consequentemente, a força axial pode ser escrita como $t=\left|\vec{t}_{a}\right|=\left|\vec{t}_{b}\right|=\sigma a$. Como se está admitindo que o elemento bi-articulado possa sofrer deformações finitas é necessário definir um modelo constitutivo hiperelástico. Neste caso, admite-se a existência da energia de deformação, $\psi$, por unidade de volume inicial, $V$, quando o elemento bi-articulado se desloca da configuração indeformada para a configuração deformada. Em qualquer instante $t$ a força axial $t(l)$ atuante no elemento depende do comprimento $l$. Se o elemento se alonga de uma pequena quantidade $d l$, então o trabalho interno é dado por $t(l) d l$ e o trabalho total por unidade de volume inicial é escrito conforme a Equação 6.

$$
\psi=\frac{1}{V} \int_{L}^{l} t(l) d l=\int_{L}^{l} \frac{\sigma a}{V} d l
$$

Levando em conta que $v=a l$ e a deformação instântanea, $d \varepsilon=d l / l$, a expressão da energia interna pode ser reescrita conforme a Equação 7.

$$
\begin{aligned}
\psi=\int_{L}^{l} \frac{\sigma a}{V} d l= & \int_{L}^{l} \frac{v}{V} \sigma \frac{d l}{l}=\int_{L}^{l} J \sigma d \varepsilon \\
& =\int_{L}^{l} \tau d \varepsilon \\
\tau & =J \sigma
\end{aligned}
$$


Em que $\tau$ é denominada tensão de Kirchhoff. Nota-se que esta tensão é energeticamente conjugada com a deformação instantânea porque define a quantidade de energia de deformação armazenada no elemento por unidade de volume inicial através do trabalho $\tau \times$ $d \varepsilon$.

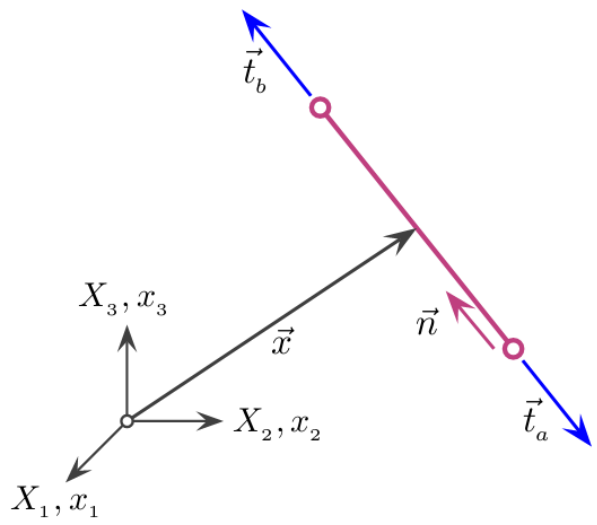

FIGURA 2: Forças internas do elemento de barra biarticulado 3D.

FONTE: Próprios autores.

Assume-se que o módulo de Young $E$ é dado pelo gradiente da tensão de Kirchhoff $\tau$ em relação a deformação logarítmica $\varepsilon$, isto é, $E=$ $d \tau / d \varepsilon$ ou $d \tau=E d \varepsilon$, e que $E$ seja constante. Portanto, pode-se escrever que

$$
\begin{gathered}
\int_{0}^{\tau} d \tau=E \int_{L}^{l} \frac{d l}{l} \\
\tau=E \ln \lambda
\end{gathered}
$$

Note que novamente a deformação logarítmica, ln $\lambda$, surge naturalmente como integral da deformação instantânea, $d \varepsilon$. Substituindo a equação (8) na equação (7) obtém-se a energia de deformação por unidade de volume inicial como

$$
\begin{gathered}
\psi=E \int_{L}^{l} \ln \left(\frac{l}{L}\right) \frac{d l}{l}=\frac{1}{2} E(\ln \lambda)^{2} \Rightarrow \tau \\
=\frac{d \psi}{d(\ln \lambda)}
\end{gathered}
$$

Frequentemente, a equação (9) é utilizada para definir materiais hiperelásticos. Se o material for incompressível, $v=1 / 2$ e $J=1$, chega-se a relação $\sigma=E \ln \lambda$. Por fim, utilizando as relações $v=a l, J=v / V, \sigma=(1 / J) \tau$ e $\tau=E \ln \lambda$, as forças internas podem ser reescritas como

$$
\begin{gathered}
\vec{t}_{b}=\frac{1}{J} \tau a \vec{n}=\frac{V}{v} \tau \frac{v}{l} \vec{n}=\frac{V E}{l} \ln \lambda \vec{n} \\
=\lambda^{-1} E A \ln \lambda \vec{n} \\
\vec{t}_{a}=-\vec{t}_{b}
\end{gathered}
$$

\section{MATRIZ DE RIGIDEZ TANGENTE}

A matriz de rigidez tangente do elemento biarticulado é obtida através da derivada direcional do vetor de forças internas com relação a posição atual $\vec{x}$, na direção dos deslocamentos incrementais das extremidades do elemento, isto é, $D \vec{t}^{(e)}\left(\vec{x}^{(e)}\right)\left[\vec{u}^{(e)}\right]=[K]_{T}^{(e)} \vec{u}^{(e)}$. Partindo das forças internas atuantes em uma das extremidades do elemento e a deformação axial do mesmo, que são expressos por $\quad \vec{t}_{b}=\tau \frac{V}{l} \vec{n} \quad$ e $\quad \varepsilon=\ln \left(\frac{l}{L}\right)$, respectivamente, e, além disso, utilizando as equações (1), (3), (4) e (5), obtém-se a matriz de rigidez tangente de acordo com os passos algébricos mostrados a seguir

$$
\begin{gathered}
D \vec{t}_{b}(\vec{x})[\vec{u}]=D \tau(\vec{x})[\vec{u}] \frac{V}{l} \vec{n}+\tau D\left(\frac{V}{l}(\vec{x})\right)[\vec{u}] \vec{n}+\tau \frac{V}{l} D \vec{n}(\vec{x})[\vec{u}] \\
=\frac{d \tau}{d \varepsilon} D \varepsilon(\vec{x})[\vec{u}] \frac{V}{l} \vec{n}+\tau D\left(\frac{V}{l}(\vec{x})\right)[\vec{u}] \vec{n}+\tau \frac{V}{l} D \vec{n}(\vec{x})[\vec{u}] \\
=\frac{d \tau}{d \varepsilon} \frac{1}{l} \vec{n} \cdot\left(\vec{u}_{b}-\vec{u}_{a}\right) \frac{V}{l} \vec{n}+\tau V D\left(l^{-1}(\vec{x})\right)[\vec{u}] \vec{n}+ \\
+\tau \frac{V}{l}\left(D\left(l^{-1}(\vec{x})\right)[\vec{u}]\left(\vec{x}_{b}-\vec{x}_{a}\right)+\frac{1}{l} D\left(\vec{x}_{b}-\vec{x}_{a}\right)[\vec{u}]\right) \\
=\frac{d \tau}{d \varepsilon} \frac{V}{l^{2}} \vec{n} \cdot\left(\vec{u}_{b}-\vec{u}_{a}\right) \vec{n}+2 \tau V D\left(l^{-1}(\vec{x})\right)[\vec{u}] \vec{n}+\frac{\tau V}{l^{2}}\left(\vec{u}_{b}-\vec{u}_{a}\right) \\
=\frac{d \tau}{d \varepsilon} \frac{V}{l^{2}} \vec{n} \cdot\left(\vec{u}_{b}-\vec{u}_{a}\right) \vec{n}-\frac{2}{l^{2}} \tau V \vec{n}\left(\vec{u}_{b}-\vec{u}_{a}\right) \vec{n}+\frac{\tau V}{l^{2}}\left(\vec{u}_{b}-\vec{u}_{a}\right) \\
=\left(\frac{d \tau}{d \varepsilon} \frac{V}{l^{2}}-\frac{2 \tau V}{l^{2}}\right) \vec{n} \cdot\left(\vec{u}_{b}-\vec{u}_{a}\right) \vec{n}+\frac{\tau V}{l^{2}}\left(\vec{u}_{b}-\vec{u}_{a}\right) \\
=\left(\frac{d \tau}{d \varepsilon} \frac{V}{l^{2}}-\frac{2 \tau V}{l^{2}}\right)[\vec{n} \otimes \vec{n}]_{3 \times 3}\left(\vec{u}_{b}-\vec{u}_{a}\right)+\frac{\tau V}{l^{2}}[I]_{3 \times 3}\left(\vec{u}_{b}-\vec{u}_{a}\right) \\
=\left(\frac{d \tau}{d \varepsilon} \frac{V}{v} \frac{2 \sigma a}{l}-\frac{2 \sigma a}{l}\right)[\vec{n} \otimes \vec{n}]_{3 \times 3}\left(\vec{u}_{b}-\vec{u}_{a}\right)+\frac{\sigma a}{l}[I]_{3 \times 3}\left(\vec{u}_{b}-\vec{u}_{a}\right)
\end{gathered}
$$


Observe que a penúltima expressão da equação (11) está escrita em função da tensão de Kirchhoff, enquanto que a última expressão está expressa em função da tensão verdadeira de Cauchy. Lembrando que $D \vec{t}_{a}(\vec{x})[\vec{u}]=-D \vec{t}_{a}(\vec{x})[\vec{u}]$ e após um rearranjo em forma matricial dos termos da equação (11), chega-se a

$$
\begin{aligned}
D \vec{t}^{(e)}\left(\vec{x}^{(e)}\right)\left[\vec{u}^{(e)}\right] & =[K]_{T}^{(e)} \vec{u}^{(e)} \\
& =\left[\begin{array}{ll}
K_{a a} & K_{a b} \\
K_{b a} & K_{b b}
\end{array}\right]\left\{\begin{array}{l}
\vec{u}_{a} \\
\vec{u}_{b}
\end{array}\right\}
\end{aligned}
$$

onde $\quad[K]_{a a}=[K]_{b b}=k[\vec{n} \otimes \vec{n}]_{3 \times 3}+\frac{t}{l}[I]_{3 \times 3}$; $[K]_{a b}=[K]_{b a}=-[K]_{b b}, k$ é o coeficiente de rigidez do elemento de barra biarticulado e, $t=\sigma a$, é a força axial atuante no elemento. $O$ coeficiente de rigidez $k$ requer o cálculo do modulo elástico tangente, que neste trabalho assume-se um valor constante dado por $\frac{d \tau}{d \epsilon}=E$. Portanto, pode-se escrever a seguinte expressão para o coeficiente de rigidez $k$

$$
\begin{aligned}
k=\left(\frac{d \tau}{d \varepsilon} \frac{V}{v} \frac{a}{l}-\right. & \left.\frac{2 t}{l}\right)=\left(\frac{V}{v} \frac{E a}{l}-\frac{2 t}{l}\right) \\
= & \frac{V}{l^{2}}(E-2 \tau) \\
& =\lambda^{-1} \frac{A}{l}(E-2 \tau)
\end{aligned}
$$

\section{COMPORTAMENTO ELASTOPLÁSTICO}

Neste item, estende-se a formulação anterior para incluir as deformações permanentes no elemento de barra biarticulado. Admite-se que o material do elemento apresenta um comportamento elastoplástico com endurecimento isotrópico. Para maiores detalhes consultar o capítulo 1 da referência Simo e Hughes (1998). Neste trabalho discute-se com mais detalhes a decomposição multiplicativa do estiramento do elemento para simular deformações plásticas finitas e sua implementação computacional. No Quadro 2 detalha-se os principais conceitos da teoria da plasticidade para o estado unidimensional de tensão e com deformações infinitesimais.

\section{QUADRO 2: Plasticidade unidimensional com endurecimento isotrópico.}

i. Relação tensão-deformação

$\tau=E\left(\varepsilon-\varepsilon_{p}\right)$

ii. Regra de fluxo plástico e lei de endurecimento isotrópico

$\dot{\varepsilon}_{p}=\gamma \operatorname{sign}(\tau)$

$\dot{\alpha}=\gamma$

iii. Critério de escoamento

$f(\tau, \alpha)=|\tau|-\left(\tau_{y}+H \alpha\right) \leq 0$

iv. Condições de complementariedade de Kuhn-Tucker

$\gamma \geq 0, f(\tau, \alpha) \leq 0, \gamma f(\tau, \alpha)=0$

v. Condição de consistência

$\gamma f(\tau, \alpha)=0($ se $f(\tau, \alpha)=0)$ 


\subsection{DECOMPOSIÇÃO MULTIPLICATIVA DO ESTIRAMENTO}

Suponha que no instante atual $t$ a tensão de Kirchhoff no elemento de barra bi-articulado tenha ultrapassado o limite de escoamento do material $\tau_{Y}$. Neste instante 0 estiramento do elemento é dado por $\lambda=\frac{l}{L}$. Agora, admita-se que a força axial atuante no elemento seja retirada, portanto, uma parte da deformação será recuperada elasticamente enquanto que a outra parte será permanente no elemento, cujo comprimento será dado por $l_{p}$. De acordo com a Figura 3, define-se como estiramento elástico a relação $\lambda_{e}=\frac{l}{l_{p}}$, e como estiramento plástico a relação $\lambda_{p}=\frac{l_{p}}{L}$. Por fim, adota-se uma decomposição multiplicativa do estiramento da barra em uma parte elástica e em uma parte plástica, tal que, $\lambda=\lambda_{e} \lambda_{p}$.

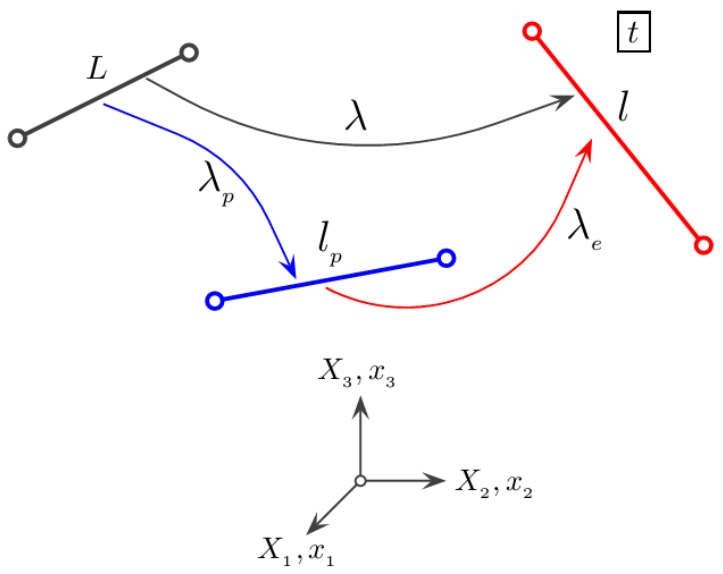

FIGURA 3: Decomposição multiplicativa do estiramento do elemento de barra biarticulado 3D. FONTE: Próprios autores.

Como já foi dito, a configuração descarregada tem um comprimento $l_{p}$, que pode ser vista como uma nova configuração a partir da qual o elemento é estirado elasticamente até a configuração atual, definida pela coordenada $\vec{x} \mathrm{e}$ com comprimento $l$. Este estado referência alternativo, é denominado de configuração inelástica ou plástica. Ao aplicar a função logarítmica no estiramento $\lambda$ atual da barra, surge naturalmente uma decomposição aditiva entre as componentes elástica e plástica da deformação logarítmica, que se expressa como

$$
\left\{\begin{array}{c}
\ln \lambda=\ln \left(\lambda_{e} \lambda_{p}\right)=\ln \lambda_{e}+\ln \lambda_{P} \\
\varepsilon=\ln \lambda \quad \varepsilon_{e}=\ln \lambda_{e} \quad \varepsilon_{p}=\ln \lambda_{p} \\
\varepsilon=\varepsilon_{e}+\varepsilon_{p}
\end{array}\right.
$$

Observe que a última expressão da equação (14) corresponde à decomposição aditiva empregada na teoria de deformações plásticas infinitesimais, conforme descrita no Quadro 2. Neste trabalho, adota-se um esquema de integração implícito para integrar o modelo de plasticidade finita. Portanto, torna-se necessário a definição das variáveis cinemáticas em pontos discretos do domínio no tempo, isto é, levar em conta o incremento destas variáveis ao longo do tempo. Portanto, de acordo com a Figura 4, considera-se um passo de tempo $\Delta t$ durante o qual a barra se move da posição $n$ no instante $t$ para a posição $n+1$ no instante $t+\Delta t$. Os comprimentos da barra nos instantes $t$ e $t+\Delta t$ são $l_{n}$ e $l_{n+1}$, respectivamente. A cada incremento de tempo, isto é, entre os instantes $t$ e $t+\Delta t$ podem ser definidas as configurações descarregadas da barra, que são definidas por $l_{p, n}$ e $l_{p, n+1}$, respectivamente. Caso não haja deformações plásticas neste intervalo de tempo então, consequentemente, $l_{p, n}=l_{p, n+1}$.

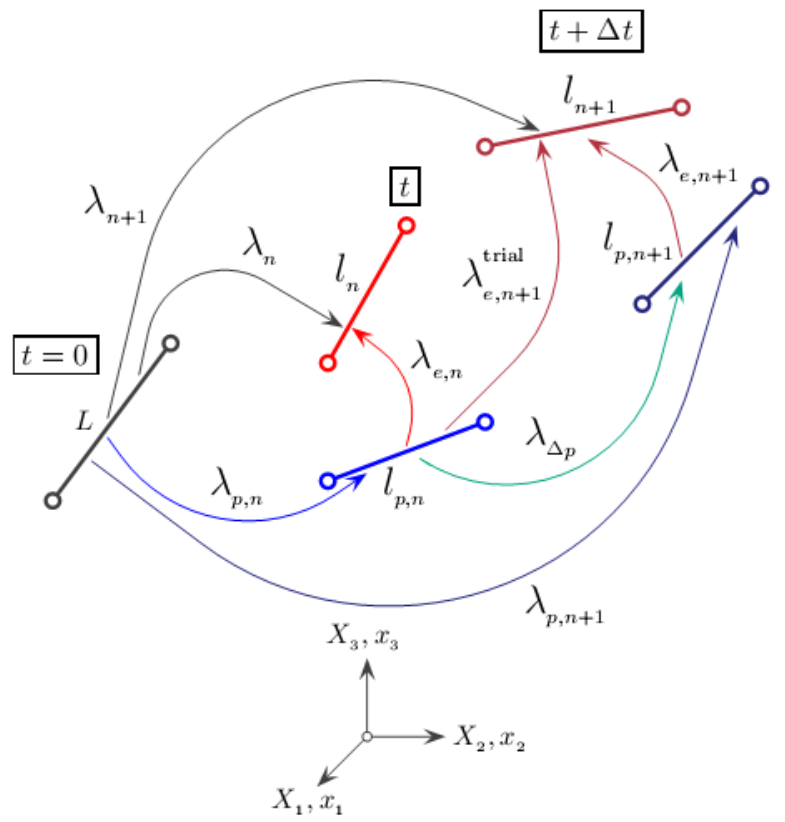

FIGURA 4: Valores discretos das variáveis cinemáticas do elemento de barra biarticulado.

FONTE: Próprios autores. 
De acordo com a Figura 4, podem-se definir os seguintes estiramentos: 1) Entre as configurações indeformada e deformada no instante $\left.t, \lambda_{n}=\lambda_{e, n} \lambda_{p, n}=\frac{l_{n}}{l_{p}} \frac{l_{p}}{L}=\frac{l_{n}}{L} ; 2\right)$ Entre as configurações indeformada e deformada no instante $\quad t+\Delta t, \quad \lambda_{n+1}=\lambda_{e, n+1} \lambda_{p, n+1}=$ $\frac{l_{n+1}}{l_{p, n+1}} \frac{l_{p, n+1}}{L}=\frac{l_{n+1}}{L}$. Como ponto de partida, na ausência de uma informação melhor, admite-se que durante o incremento de tempo $\Delta t$, o elemento de barra biarticulado sofre somente deformações elásticas, desta maneira, o estiramento $\lambda_{n+1}$ pode ser expresso como uma decomposição multiplicativa entre o estiramento plástico $\lambda_{p, n}$ definido no instante anterior $t$ e uma estimativa de estiramento elástico, tal que,

$$
\begin{aligned}
\lambda_{n+1}=\lambda_{e, n+1}^{\text {trial }} \lambda_{p, n} \Rightarrow \lambda_{e, n+1}^{\text {trial }} & =\lambda_{n+1} \lambda_{p, n}^{-1} \\
= & \frac{l_{n+1}}{L} \frac{L}{l_{p, n}}=\frac{l_{n+1}}{l_{p, n}}
\end{aligned}
$$

Aplica-se a função logarítmica no estiramento elástico definido na equação (15), para obter a decomposição aditiva das deformações, que se expressa como

$$
\begin{aligned}
\ln \left(\lambda_{e, n+1}^{\text {trial }}\right) & =\ln \left(\lambda_{n+1} \lambda_{p, n}^{-1}\right) \\
& =\ln \lambda_{n+1}-\ln \lambda_{p, n} \\
& \Rightarrow \varepsilon_{e, n+1}^{\text {trial }} \\
& =\varepsilon_{n+1}-\varepsilon_{p, n}
\end{aligned}
$$

Portanto, a estimativa para o estado de tensão no instante $t+\Delta t$ será dado pela expressão $\tau_{n+1}^{\text {trial }}=E \varepsilon_{e, n+1}^{\text {trial }}$. No Quadro 3 descreve-se o algoritmo implícito, denominado return mapping, utilizado neste trabalho para integrar o modelo de plasticidade aqui descrito. A dedução detalhada deste esquema de integração encontra-se no capítulo 1 da referência Simo e Hughes (1998).

\section{QUADRO 3: Algoritmo Return Mapping.}

i. Cálculo da tensão trial

$$
\tau_{n+1}^{\text {trial }}=E\left(\varepsilon_{n+1}-\varepsilon_{p, n}\right)
$$

ii. Cálculo do critério de escoamento trial

$$
\begin{aligned}
& f_{n+1}^{\text {trial }}=\left|\tau_{n+1}^{\text {trial }}\right|-\left(\tau_{Y}-H \alpha_{n}\right) \\
& \text { If } f_{n+1}^{\text {trial }} \leq 0 \text { then }
\end{aligned}
$$

Passo Elástico $\Rightarrow\left\{\begin{array}{c}\tau_{n+1}=\tau_{n+1}^{\text {trial }} \\ \varepsilon_{p, n+1}=\varepsilon_{p, n} \\ \alpha_{n+1}=\alpha_{n}\end{array} \Rightarrow\right.$ exit

else

Passo Plástico $\Longrightarrow\left\{\begin{array}{c}\Delta \gamma=\frac{f_{n+1}^{\text {trial }}}{(E+H)} \\ \tau_{n+1}=\tau_{n+1}^{\text {trial }}-E \Delta \gamma \operatorname{sign}\left(\tau_{n+1}^{\text {trial }}\right) \\ \varepsilon_{p, n+1}=\varepsilon_{p, n}+\Delta \gamma \operatorname{sign}\left(\tau_{n+1}^{\text {trial }}\right) \\ \alpha_{n+1}=\alpha_{n}+\Delta \gamma\end{array}\right.$

endif 


\section{EXEMPLOS NUMÉRICOS}

A seguir, dois exemplos que ilustram o comportamento não-linear tanto geométrico quanto físico serão analisados usando a formulação aqui apresentada. Para isto, será utilizado um programa de elementos finitos denominado truss_nl.f90 escrito pelo autor usando a linguagem de programação FORTRAN90. Em ambos os casos as magnitudes das deformações plásticas serão finitas. Para obter as trajetórias de equilíbrio utiliza-se um esquema incremental-iterativo com o método de Newton-Raphson em conjunção com o método de controle de deslocamentos conforme descrito no capítulo 8 da referência Crisfield (1991).

\subsection{PÓRTICO DE LEE}

Neste exemplo, analisa-se o comportamento do pórtico de Lee tanto na fase elástica quanto na fase inelástica. As características físicas e geométricas, as condições de contorno e de carregamento são mostradas na Figura $5 a$. Discretiza-se o pórtico de Lee com elementos de barra biarticulado 2D, para isto, utiliza-se uma célula com 4 nós e 6 elementos, cujas características geométricas mostram-se na Figura $5 \mathrm{~b}$. Ao todo, foram utilizadas 119 células para discretizar o pórtico, o que resultou em 240 nós e 596 elementos. $\mathrm{Na}$ análise elástica $\mathrm{o}$ pórtico sofre grandes translações e grandes rotações de corpo rígido, porém deformações infinitesimais. Na Figura $5 c$ mostram-se as trajetórias de equilíbrio resultantes da análise elástica bem como da análise elastoplástica. $\mathrm{Na}$ análise elastoplástica o pórtico apresenta deformações plásticas finitas somente nas regiões de rótulas plásticas, também, nesta fase, o pórtico apresenta grandes translações e grandes rotações de corpo rígido. Nas regiões afastadas das rótulas plásticas a deformação total é infinitesimal.

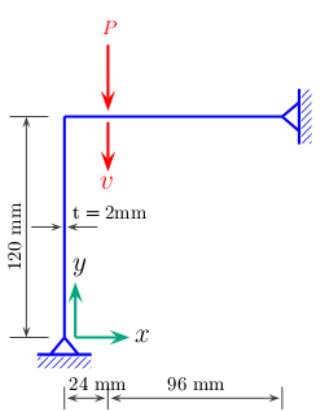

[a]

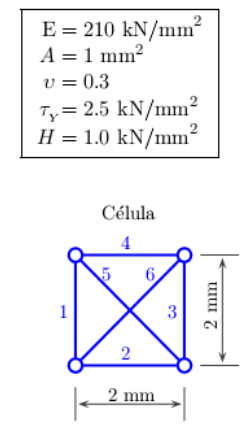

[b]

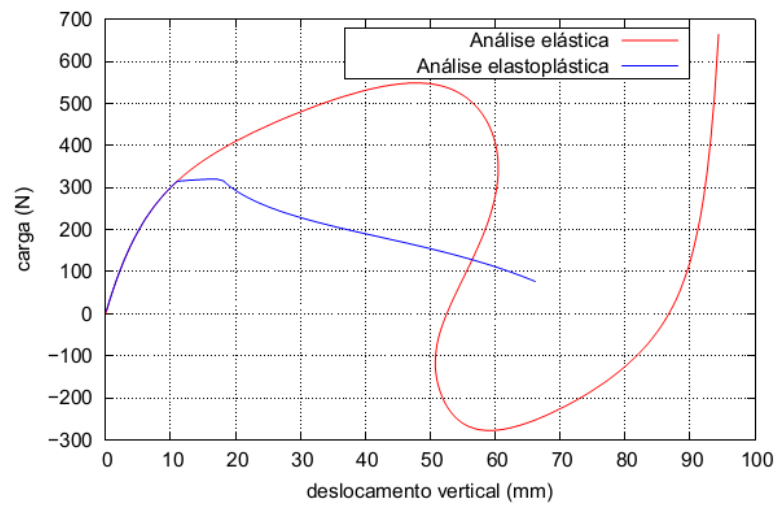

[c]

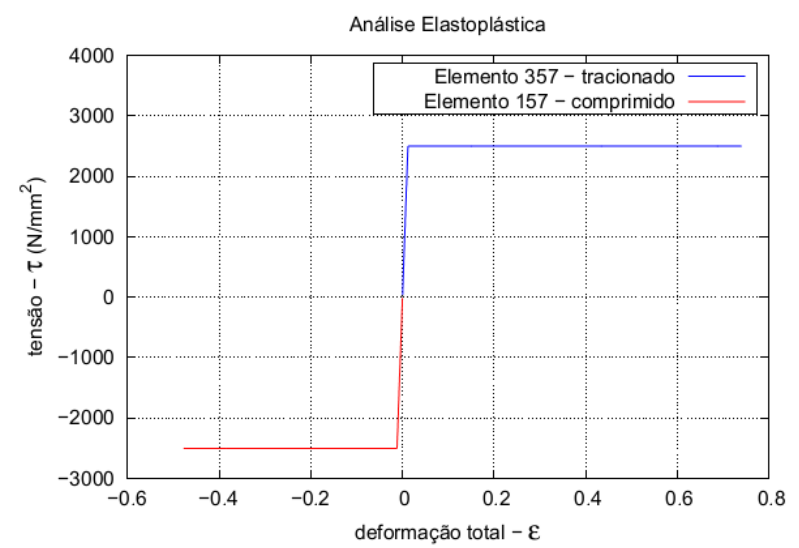

$[d]$

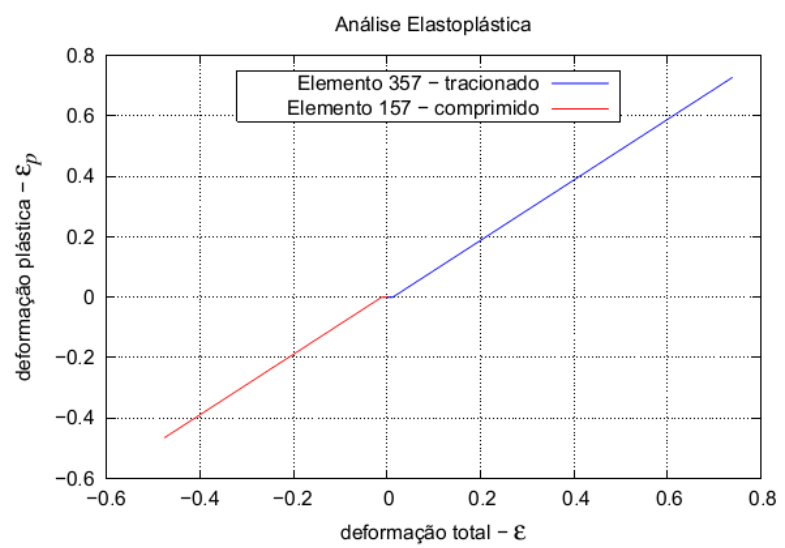

[e]

FIGURA 5: [a] Pórtico de Lee. Características físicas e geométricas. [b] Célula de elementos biarticulados 2D utilizada na discretização do pórtico. [c] Trajetória de equilíbrio. [d] Relação tensão $\times$ deformação. [e] Relação deformação plástica $\times$ deformação total. [f] Configurações de equilíbrio com deformações inelásticas. (Continua). 


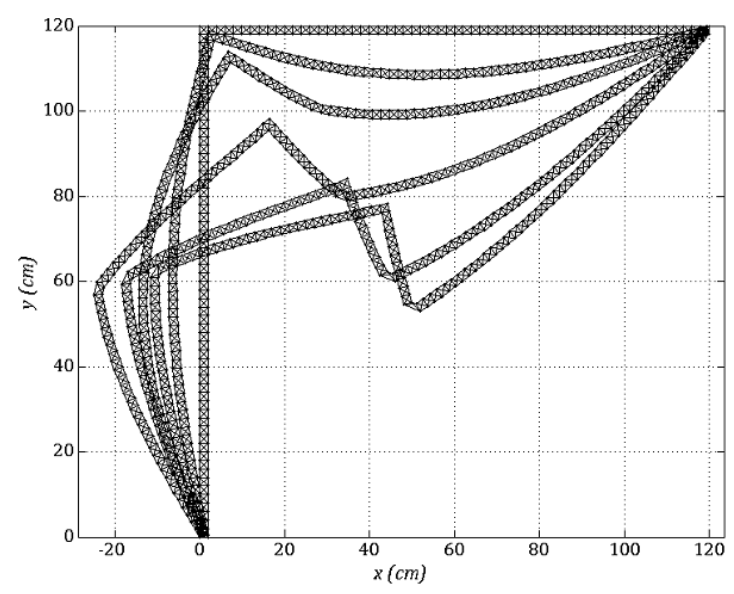

[f]

FIGURA 5: (Continuação) [a] Pórtico de Lee. Características físicas e geométricas. [b] Célula de elementos biarticulados 2D utilizada na discretização do pórtico. [c] Trajetória de equilíbrio. [d] Relação tensão $\times$ deformação. [e] Relação deformação plástica $\times$ deformação total. [f] Configurações de equilíbrio com deformações inelásticas.

FONTE: Próprios autores.

As articulações plásticas formam-se na região sob aplicação da carga e a meia altura da coluna conforme mostra-se na Figura $5 f$. Monitoraram-se alguns elementos nessas regiões com as maiores deformações plásticas. Foram monitorados o elemento 357 sujeito a tração pertencente à região sob a carga e o elemento 157 sujeito à compressão pertencente à região da metade da coluna. Na Figura $5 d$ mostram-se as relações tensões $\times$ deformações tanto para elemento 357 (tracionado) quanto para o elemento 157 (comprimido). Observa-se nesta figura que a magnitude máxima da deformação total para o elemento 357 é da ordem de 0,7 enquanto que para o elemento 157 é da ordem de 0,4. Na Figura 5e mostram-se as relações entre as deformações plásticas $\times$ deformações totais para os elementos 357 e 157, respectivamente. Observa-se nesta figura que as parcelas das deformações plásticas para ambos elementos alcançam aproximadamente um percentual entre $96 \%$ a $98 \%$ da deformação total, o que implica que as deformações plásticas em ambos elementos são finitas enquanto que as deformações elásticas nesses elementos são infinitesimais.

\subsection{VIGA 3D ENGASTADA}

Neste exemplo mostra-se o comportamento de uma viga engastada tanto na fase elástica quanto na fase elastoplástica sob deformações finitas. Este exemplo foi proposto pelos autores Bonet e Wood (2008) que utilizaram o programa FLagSHyp de autoria dos próprios autores disponível no site www.flagshyp.com. Esses autores discretizaram a viga com elementos hexaédricos de 8 nós e adotaram um modelo hiperelástico-plástico quase-incompressível cujas propriedades geométricas e mecânicas estão descritas na Figura 6a. Para modelar a viga com elementos de treliça plana foi utilizado a célula 2D, mostrada na Figura $6 b$, resultando em uma malha de 42 nós e 101 elementos. Por outro lado, para modelar a viga com elementos de treliça espacial foi utilizada a célula $3 \mathrm{D}$, também mostrada na Figura $6 \mathrm{~b}$, resultando em uma malha de 84 nós e 366 elementos. Na Figura $6 \mathrm{c}$ mostram-se as trajetórias de equilíbrio tanto para análise elástica quanto para análise elastoplástica obtidas com as células 2D e 3D, respectivamente. Observa-se que essas trajetórias de equilíbrio são coincidentes com as trajetórias obtidas por Bonet e Wood (2008).

A articulação plástica forma-se na região próxima ao engaste conforme mostra-se na Figura 6f. $\mathrm{Na}$ análise elastoplástica a viga engastada 3D apresenta deformações plásticas finitas somente na região da articulação plástica, também nesta fase, a viga apresenta grandes translações e grandes rotações de corpo rígido conforme pode-se observar na Figura 6f. Monitoraram-se alguns elementos nessas regiões com as maiores deformações plásticas. Foram monitorados 0 elemento 1 sujeito a compressão e o elemento $2 \mathrm{sob}$ 
tração utilizando células 2D. Por outro lado, utilizando células 3D foram monitorados 0 elemento 7 sujeito a compressão e o elemento 9 sob tração. Na Figura $6 \mathrm{~d}$ mostram-se as relações tensões $\times$ deformações totais para esses elementos. Observa-se que essas relações são coincidentes tanto para os elementos tracionados quanto para os elementos comprimidos indiferentemente da célula utilizada, isto é, os resultados foram idênticos utilizando células 2D bem como células 3D. Na Figura 6e mostram-se as relações deformações plásticas $\times$ deformações totais para os elementos 1 e 2 utilizando células $2 D$ e para os elementos 7 e 9 utilizando células 3D. Observa-se que os resultados são coincidentes. Observa-se nesta figura que as parcelas das deformações plásticas para ambos os elementos alcançam aproximadamente um percentual de $96 \%$ da deformação total, o que implica que as deformações plásticas nesses elementos são finitas enquanto que as deformações elásticas são infinitesimais.

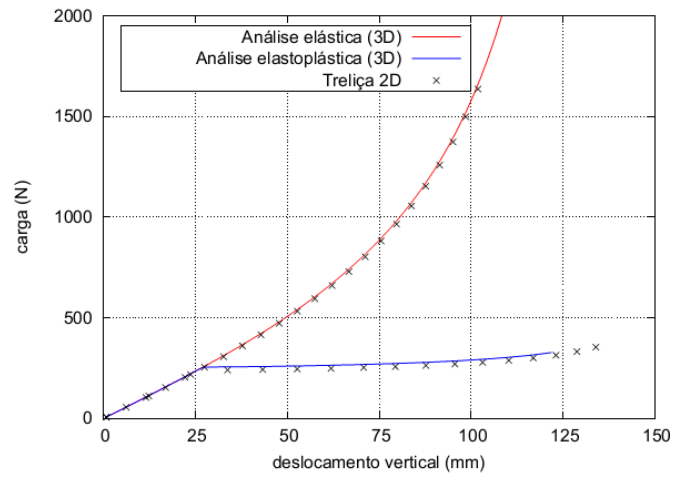

[c]
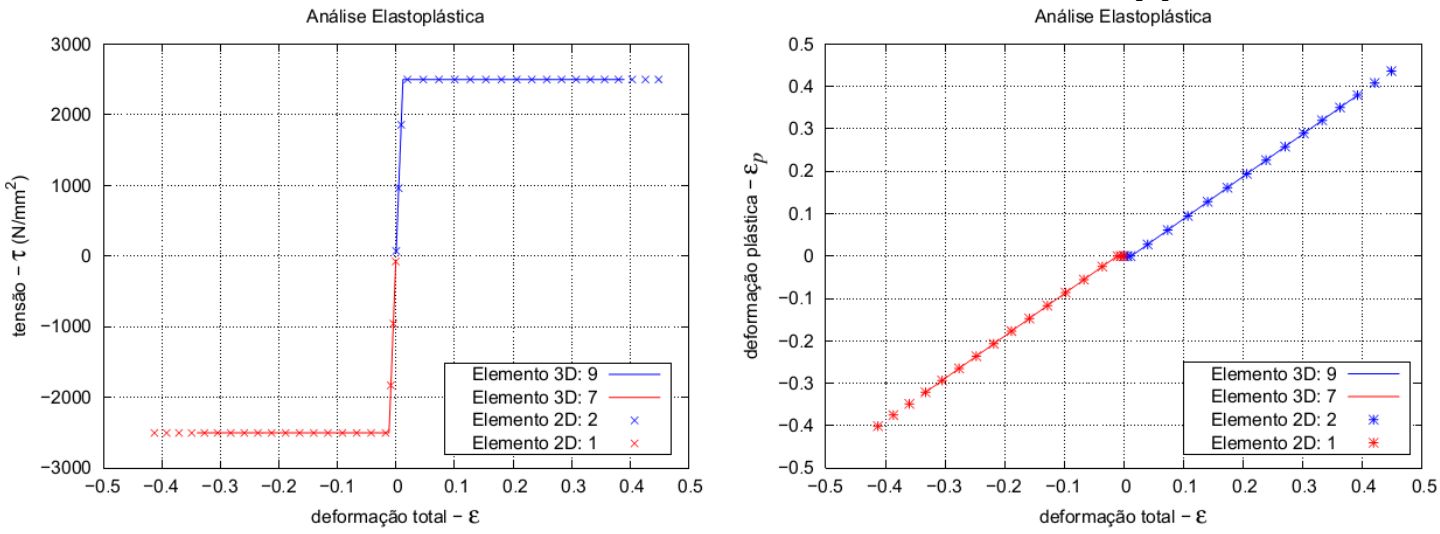

[d]

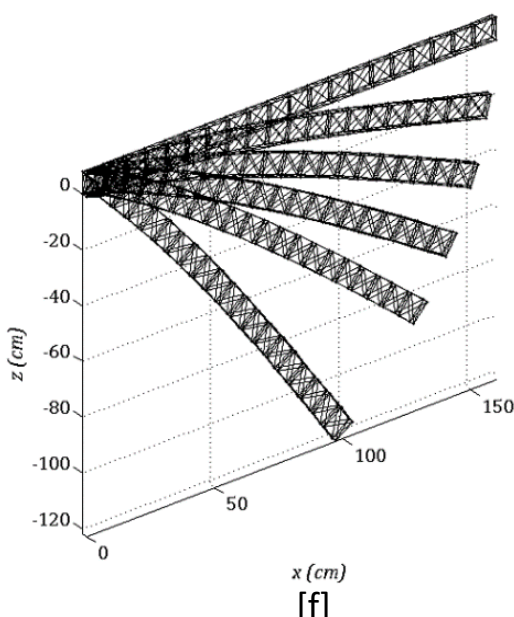

$x(\mathrm{~cm})$
$[\mathrm{f}]$

FIGURA 6: [a] Viga 3D engastada. Características físicas e geométricas. [b] Células de elementos biarticulados 2D e 3D utilizadas na discretização da viga.[c] Trajetórias de equilíbrio. [d] Relações tensões $\times$ deformações. [e] Relações deformações plásticas $\times$ deformações totais. [f] Configurações de equilíbrio com deformações inelásticas utilizando células 3D.

FONTE: Próprios autores. 
Neste exemplo mostra-se o comportamento de uma viga engastada tanto na fase elástica quanto na fase elastoplástica sob deformações finitas. Este exemplo foi proposto pelos autores Bonet e Wood (2008) que utilizaram o programa FLagSHyp de autoria dos próprios autores disponível no site www.flagshyp.com. Esses autores discretizaram a viga com elementos hexaédricos de 8 nós e adotaram um modelo hiperelástico-plástico quase-incompressível cujas propriedades geométricas e mecânicas estão descritas na Figura 6a. Para modelar a viga com elementos de treliça plana foi utilizado a célula 2D, mostrada na Figura 6b, resultando em uma malha de 42 nós e 101 elementos. Por outro lado, para modelar a viga com elementos de treliça espacial foi utilizada a célula $3 D$, também mostrada na Figura $6 \mathrm{~b}$, resultando em uma malha de 84 nós e 366 elementos. Na Figura 6c mostram-se as trajetórias de equilíbrio tanto para análise elástica quanto para análise elastoplástica obtidas com as células 2D e 3D, respectivamente. Observa-se que essas trajetórias de equilíbrio são coincidentes com as trajetórias obtidas por Bonet e Wood (2008).

A articulação plástica forma-se na região próxima ao engaste conforme mostra-se na Figura 6f. Na análise elastoplástica a viga engastada 3D apresenta deformações plásticas finitas somente na região da articulação plástica, também nesta fase, a viga apresenta grandes translações e grandes rotações de corpo rígido conforme pode-se observar na Figura 6f. Monitoraram-se alguns elementos nessas regiões com as maiores deformações plásticas. Foram monitorados 0 elemento 1 sujeito a compressão e o elemento 2 sob tração utilizando células 2D. Por outro lado, utilizando células 3D foram monitorados 0 elemento 7 sujeito a compressão e o elemento 9 sob tração. $\mathrm{Na}$ Figura $6 \mathrm{~d}$ mostram-se as relações tensões $\times$ deformações totais para esses elementos. Observa-se que essas relações são coincidentes tanto para os elementos tracionados quanto para os elementos comprimidos indiferentemente da célula utilizada, isto é, os resultados foram idênticos utilizando células 2D bem como células 3D. $\mathrm{Na}$ Figura 6e mostram-se as relações deformações plásticas $\times$ deformações totais para os elementos 1 e 2 utilizando células 2D e para os elementos 7 e 9 utilizando células 3D. Observa-se que os resultados são coincidentes. Observa-se nesta figura que as parcelas das deformações plásticas para ambos os elementos alcançam aproximadamente um percentual de $96 \%$ da deformação total, o que implica que as deformações plásticas nesses elementos são finitas enquanto que as deformações elásticas são infinitesimais.

\section{CONCLUSÕES}

Ao adotar a decomposição multiplicativa do estiramento uniaxial da barra e definir como medida de deformação a função logarítmica deste estiramento, chegou-se a uma decomposição aditiva das deformações, o que possibilitou um tratamento analítico e numérico mais simples para retratar deformações plásticas finitas, mas isto é possível somente para estados uniaxiais de tensãodeformação. Daí a razão pela qual, neste trabalho, formulou-se a cinemática e o comportamento elastoplástico de um elemento de barra biarticulado. É importante esclarecer que este trabalhou não trouxe nenhuma abordagem nova sobre o tema da plasticidade computacional, entretanto, tentou-se descrever de maneira bastante simples a formulação teórica e numérica sobre deformações finitas tanto na fase elástica quanto na fase inelástica. Quanto aos resultados das simulações numéricas obteve-se resultados qualitativos que expressam o comportamento mecânico das estruturas sob grandes translações, grandes rotações e deformações finitas. Isto é, do ponto de vista qualitativo, pode-se discretizar sólidos 2D e 3D utilizando elementos de barra biarticulados 2D e 3D, respectivamente. Talvez, o principal atrativo desses elementos é retratar temas de maior complexidade de uma maneira mais palatável e de fácil entendimento.

\section{AGRADECIMENTOS}

Os autores gostariam de agradecer pelo apoio a esta e outras pesquisas à: A Faculdade de Tecnologia da Universidade de Brasília; ao Programa 
de Pós-Graduação em Estruturas de Construção (PECC); A Faculdade de Engenharia da Universidade Federal de Goiás - Regional Catalão; ao Programa de Pós-Graduação em Modelagem e Otimização (POSMOT) - IMTec; ao Instituo Federal de Brasília; e às Agências de fomento CNPq e CAPES.

\section{REFERÊNCIAS BIBLIOGRÁFICAS}

BELYTSCHKO, T.; LIU W, K.; MORAN, B. Nonlinear finite elements for continua and strucutures. John Wiley, 2000.

BONET, J.; WOOD, R.D. Nonlinear continuum mechanics for finite element analysis. 2nd Edition, Cambridge University Press, 2008.

BORST, R.; CRISFIELD M.A., REMMERS J.J.C e VERHOOSEL C.V. Non-linear finite element analysis of solids and structures. 2nd Edition, John Wiley, 2012.

CRISFIELD, M.A. Non-linear finite element analysis of solids and structures. Volume 1: Essentials, John Wiley, 1991.

CRISFIELD, M.A. Non-linear finite element analysis of solids and structures. Volume 2: Advanced Topics, John Wiley, 1997.

DOYLE, J.F. Nonlinear analysis of thin-walled structures. Statics, Dynamics and Stability, Springer, 2001.

HASHIGUCHI, K.; YAMAKAWA, Y. Introduction to finite strain theory for continuum elasto-plasticity. John Wiley, 2013.

KOJIĆ, M.; BATHE, K.J. Inelastic analysis of solids and structures. Springer, 2005.

KRENK, S. Non-linear modeling and analysis of solids and structures. Cambridge University Press, 2009.

NETO, E.A.S; PERIĆ, D.; OWEN, D.R.J. Computational methods for plasticity. Theory and aplications. John Wiley, 2008.

SIMO, J.C.; HUGHES, T.J.R. Computational Inelasticity. Springer, 1998.

VOYIADJIS, G.Z.; WOELKE, P. Elasto-plastic and damage analysis of plates and shells. Springer, 2010.

WRIGGERS, P. Nonlinear finite element methods. Springer, 2008.
WRIGGERS, P. Computational contact mechanics. 2nd Edition, Springer, 2002. 